\title{
A INFLUÊNCIA DA GESTÃO DE RESÍDUOS SÓLIDOS NA QUALIDADE AMBIENTAL E NAS DESIGUALDADES SOCIOESPACIAIS DE MARINGÁ-PR
}

\author{
KELLY CRISTINA RIGOLDI \\ Mestranda em Geografia \\ Universidade Estadual de Maringá ${ }^{1}$ \\ kellyrigoldi@gmail.com
}

\section{VALÉRIA LIMA}

\section{Doutora em Geografia, Docente do Departamento de Geografia da Universidade Estadual de Maringá ${ }^{1}$ \\ vlima@uem.br}

\begin{abstract}
RESUMO: O aumento da produção de resíduos sólidos decorrente da intensificação do consumo é um dos responsáveis pelos impactos ambientais e sociais nas cidades. A qualidade ambiental de um determinado local, se refere a condição positiva ou negativa deste a partir de indicadores sociais e ambientais, assim, essa é refletida também na qualidade de vida de uma população. Um fator relacionado com a qualidade ambiental é a vulnerabilidade social. Desse modo, o objetivo desse artigo é apresentar a análise do Plano de Gestão Integrada dos Resíduos Sólidos de Maringá e dos dados da coleta seletiva disponibilizados pela Secretaria de Serviços Públicos da cidade, e identificar os impactos gerados pela ineficiência de planos e sua influência da qualidade ambiental. A partir das análises identificamos que em Maringá, as áreas de exclusão social estão associadas as de baixa qualidade ambiental.
\end{abstract}

Palavras-chave: Resíduos sólidos; Coleta seletiva; Políticas públicas; Qualidade ambiental; Exclusão social.

\section{THE INFLUENCE OF SOLID WASTE MANAGEMENT ON ENVIRONMENTAL QUALITY AND SOCIO-SPATIAL INEQUALITIES IN MARINGÁ - PR}

ABSTRACT: The increase in production of solid waste resulting from the intensification of consumption is one of the responsibles for the environmental and social impacts in cities. The environmental quality of a given location, refers to a positive or negative condition based on social and environmental indicators, thus, this is also reflected in the quality of life of a population. A factor related to environmental quality is social vulnerability. In this way, the objective of this article is to present an analysis of the Integrated Management Plan for Solid Waste in Maringá and data from the selective collection made available by the city's Public Services Department, and to identify the impacts generated by the inefficiency of plans and their influence on environmental quality. . Based on the analyzes it was possible to identify that in Maringá, the areas of social exclusion are related to low environmental quality.

Keywords: Solid waste; Selective collect; Public policy; Environmental Quality; Social exclusion.

\section{LA INFLUENCIA DE LA GESTIÓN DE RESIDUOS SÓLIDOS EN LA CALIDAD AMBIENTAL Y LAS DESIGUALDADES SOCIOESPACIALES EN MARINGÁ - PR}

RESUMEN: El aumento en la producción de residuos sólidos como resultado de la intensificación del consumo es uno de los responsables del impacto ambiental y social en las ciudades. La calidad ambiental de una ubicación se refiere a su condición positiva o negativa basada en indicadores sociales y ambientales, así, esto también se refleja en la calidad de vida de una población. Un factor relacionado con la calidad ambiental es la vulnerabilidad social. Por lo tanto, el objetivo de este artículo es presentar el análisis del Plan de Manejo Integrado de Residuos Sólidos en Maringá y los datos de recolección selectiva disponibles por el Departamento de Servicios Públicos de la ciudad, e identificar los impactos generados por la ineficiencia de los planes y su influencia en la calidad ambiental. Del análisis, identificamos que, en Maringá, las áreas de exclusión social están asociadas con una baja calidad ambiental.

Palabras clave: Residuos sólidos; Recogida selectiva; Políticas públicas; Calidad del medio ambiente; Exclusión social.

${ }^{1}$ Endereço para correspondência: Av. Colombo, 5790 - Jd. Universitário, CEP: 87020-900 - Maringá-PR - Brasil. 


\section{Introdução}

O crescimento da produção de resíduos sólidos é resultado da acentuação do consumo que se manifesta como agente hegemônico na dinâmica espacial da sociedade capitalista. Entretanto, atrelado à produção de resíduos sólidos surgem os impactos ambientais e sociais, interferindo de maneira significativa na qualidade ambiental.

Por sua vez, a qualidade ambiental está intrinsicamente associada a realidade social de determinado espaço, que através de análise das interações físicas, sociais e biológicas, pode-se mensurar a condição positiva ou negativa daquele local. Desse modo, as relações existentes no espaço refletem sobre a qualidade ambiental.

A acentuação da produção de bens materiais a partir da segunda metade do século XX no Brasil, refletiram também na produção de resíduos sólidos e principalmente em relação a disposição final destes, isto atuou como alavanca para os problemas ambientais no país.

A intensificação da produção de resíduos gera impactos podendo estar vinculados a uma gestão inadequada, em muitos casos direcionada por interesses políticos. O presente artigo tem a finalidade de discutir a importância da gestão dos resíduos sólidos na cidade de Maringá/PR e como as políticas públicas ineficazes podem acentuar os problemas ambientais e sociais.

Conforme o Instituto Paranaense de Desenvolvimento Econômico e Social IPARDES (2012) Maringá/PR se localiza na mesorregião norte central Paranaense e tem uma importância estadual e regional do ponto de vista econômico e social. Possui uma estimativa populacional de $398^{2}$ mil habitantes na área urbana (Figura 1).

Figura 1 - Localização do Município de Maringá - PR

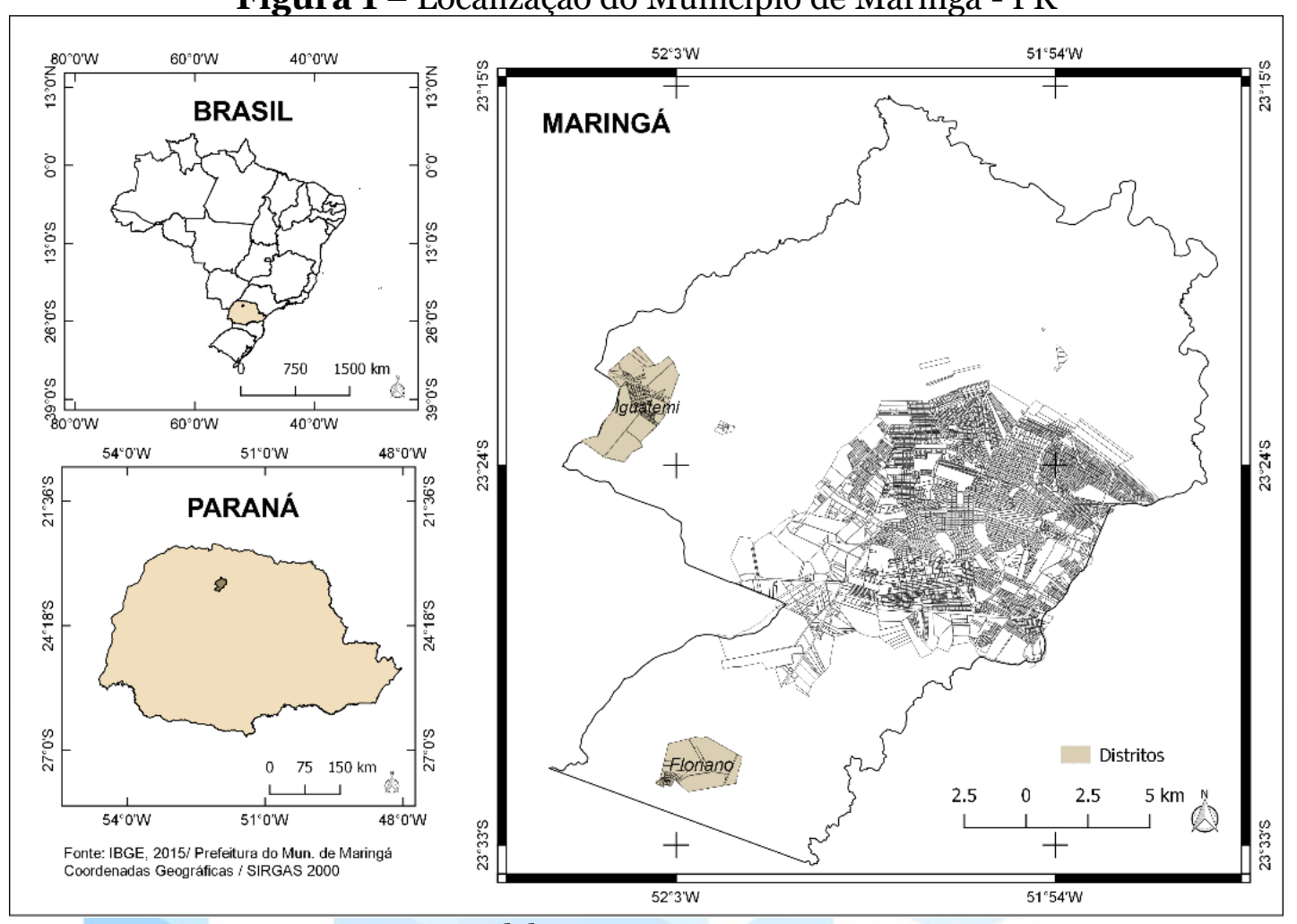

Elaboração: As autoras.

${ }^{2}$ População urbana estimada em 2017 com base nos dados IBGE de 1991, 2000 e 2001.

Kelly Cristina Rigoldi e Valéria Lima. A influência da gestão de resíduos sólidos na qualidade ambiental e nas desigualdades (...) Brazilian Geographical Journal: Geosciences and Humanities research medium,

Ituiutaba, v. 11, n. 1, p. 85-96, jan./jul. 2020. 
De acordo a Secretaria Municipal de Serviços Públicos (SEMUSP, 2017), a cidade possui dois tipos de coleta de resíduos sólidos, sendo a convencional responsável pela coleta de rejeitos os quais são destinados para o aterro sanitário da Pedreira Ingá, e a seletiva que é responsável pela coleta dos recicláveis. Maringá tem uma produção média de $285^{3}$ toneladas de resíduos sólidos diários. Atualmente, a gestão é regida pelo Plano Municipal de Gestão de Resíduos Sólidos Urbanos (PMGIRSU) estabelecido em 2017, o qual determinou estratégias e propostas de melhorias, principalmente para o programa de coleta seletiva.

\section{Procedimentos metodológicos}

As análises apresentadas neste artigo foram baseadas nos dados das coletas seletiva e convencional de resíduos sólidos de Maringá no período de 2015 a 2017. Estes dados foram disponibilizados pela SEMUSP exclusivamente para a pesquisa que foi base para este artigo, sendo os números referente a quantidade de resíduos coletados diariamente, e no caso dos recicláveis a quantidade distribuída entre cada cooperativa de reciclagem.

Para compreender a relação da quantidade de resíduos sólidos e recicláveis coletados, foi realizada estimativa da população urbana para os anos de 2015 a 2017 com base nos dados dos censos demográficos do IBGE (Instituto Brasileiro de Geografia e Estatística) de 1991, 2000 e 2010. Este foi realizado a partir de uma análise de média aritmética que apresentaram proporção de $98 \%$ e $2 \%$ respectivamente entre a população urbana e rural, a partir do teste de normalidade Jarque-Bera, o qual apresentou estimativas baixas de desvio padrão e variância, e distribuição normal dos dados com $\mathrm{p}<0,05$.

Para identificar e espacializar as áreas de disposição irregular de resíduos sólidos na cidade foram realizados trabalhos de campo com base num levantamento prévio de áreas propícias a descarte de resíduos, como terrenos vazios e fundos de vale. Para o mapeamento utilizou-se um SIG (Sistemas de Informação Geográfica) gratuito, o Q GIS na versão 2.18.23 (QGIS, 2019).

Foram utilizadas também as informações elaboradas pelo projeto "Mapeamento da exclusão social nas cidades médias do interior do Paraná", realizado pelo Grupo de Estudos e Pesquisas "Sociedade, Ambiente e Geotecnologias". Neste caso os dados de Maringá que aponta a condição de inclusão ou exclusão social a partir das variáveis: renda familiar (chefes de família sem rendimento, chefes de família com até dois salários mínimos, chefes de família com rendimento superior a 20 salários mínimos), escolaridade (analfabetos de 10 a 14 anos, chefes de família analfabetos), condições do meio em que vivem (domicílios sem banheiro, domicílios com quatro banheiros ou mais, domicílios ligados a rede de esgoto), assim como os de características demográficas (habitantes por domicílio e chefes de família de 10 a 19 anos).

\section{Qualidade ambiental urbana}

A qualidade ambiental compreende a real condição ambiental de determinado local e se estabelece a partir de correlações entre indicadores sociais, de infraestrutura e ambientais, os quais convergem para compreender a dinâmica existente no espaço geográfico.

Desse modo, compreende-se que o termo ambiental utilizado neste artigo, de acordo com Mendonça (2004), não remete somente a aspectos físicos, mas também os sociais, culturais e econômicos que, conforme Rodrigues (1998), compõem os processos da vida e de

3 Dados disponibilizados pela SEMUSP em 2017.

Kelly Cristina Rigoldi e Valéria Lima. A influência da gestão de resíduos sólidos na qualidade ambiental e nas desigualdades (...) Brazilian Geographical Journal: Geosciences and Humanities research medium, Ituiutaba, v. 11, n. 1, p. 85-96, jan./jul. 2020 Página $\mid 87$ 
sua reprodução. Assim, a qualidade ambiental se relaciona com aspectos da qualidade de vida da população.

As interações entre as atividades humanas e as características físicas do espaço se estreitam e intensificam nas cidades. É natural do homem se apropriar e gerar modificações no meio de acordo com suas necessidades. A partir das revoluções industriais, essa dinâmica ficou mais acentuada, de modo que as apropriações do espaço ocorreram de maneira mais intensa.

Mendonça (2012) defende que essa intensificação da produção e consumo dos bens materiais a partir do processo de industrialização, atuou como alimento para o desenvolvimento dos problemas ambientais urbanos, assim como o aumento populacional nas cidades a partir da segunda metade do século XX, associados à política consumista que intensificaram de maneira exponencial para o crescimento dos mesmos.

Na mesma premissa, Leff (2002) defende que a problemática ambiental surge a partir da pressão presente no crescimento populacional com a maximização dos lucros e acentuação do consumo.

Seguindo a ótica de Marques (2005), o espaço urbano sendo palco das aglomerações humanas, deve possuir infraestrutura adequada com o objetivo de atender as necessidades básicas da população e ainda evitar ou minimizar os impactos ambientais e sociais que ali possam se desenvolver. Para Martinuci (2008), a privação de acessos básicos e essenciais à população lhes retira a plena vivência como cidadãos. Os problemas ambientais urbanos estão associados ao planejamento e gestão de serviços públicos das cidades que, em muitos casos, podem ser adequados para algum interesse específico ou apenas para uma parcela da sociedade.

No que se refere aos resíduos sólidos, muitos impactos gerados se consolidam em decorrência da inadequada gestão, comprometendo a qualidade ambiental, com influência na qualidade do solo, água e ar, e em questões sanitárias.

Comumente as áreas afetadas pela baixa qualidade ambiental em decorrência da ausência ou ineficácia de serviços públicos, são também locais com presença de problemas sociais. Nesse sentido, dentro do espaço urbano é possível identificar áreas dispares em relação ao acesso a infraestrutura e serviços públicos, característica essa que compõe um cenário de desigualdade socioespacial.

\section{A desigualdade socioespacial}

A cidade, de acordo com Santos (2009), se tornou um "pólo de pobreza" ou a periferia no polo, sem poder monetário para acompanhar e se inserir nas porções mais centrais da cidade, a população de baixa renda é repelida para as regiões mais periféricas da malha urbana.

Atualmente, esse processo é bastante visível em decorrência, principalmente, do alto valor da terra nas áreas mais centrais das cidades, bem como da construção de bairros populares nas periferias urbanas com infraestrutura deficitárias, onde muitas vezes, a população não tem acesso ao atendimento à saúde, ao esgotamento sanitário, a coleta de resíduos, entre outros. Segundo Dantas, Dantas e Dias (2016), é a partir da valorização da terra que as classes mais abastadas socialmente ocupam os bairros centrais da cidade em detrimento da expulsão da população de baixa renda, as quais ocupam cada vez mais as periferias urbanas.

Conforme Mendonça (2004), diante desse contexto as classes sociais mais afetadas acabam sendo impelidas a criar uma 'cidade informal', ou seja, que anseia por infraestrutura e acessos básicos dentro dos limites legais da 'cidade formal'. Esse processo é também abordado e denominado por Lefebvre (2001) como relação centralidade-periferia, onde o urbano periférico, não se enquadra somente no conceito do termo, mas também no sentido 
de estar a margem dos acessos, ou seja, um urbano desurbarnizado, carente de uma realidade urbana.

Martinuci (2008), classifica ainda que os indivíduos ao serem privados de acessos básicos e essenciais, como saúde, educação, saneamento básico, justiça, culturais e religiosos, são consequentemente privados de sua vivencia plena como cidadãos.

Diante da atual conjuntura, Harvey (2012) alega que a qualidade de vida passou a ser uma mercadoria, assim como o direito a cidade, e a experiência urbana contemporânea recebe a classificação de liberdade, mas somente a partir daqueles que detém condições econômicas para acessá-la.

Harvey (2012) ainda defende a formação de "microestados" dentro da cidade, esses compreendem os processos de divisões que surgem dentro das áreas urbanas, onde encontramos locais totalmente dispares, de um lado o que é chamado de "vizinha riquíssima" que possui todos os tipos de serviços, e a qualidade de vida é um recurso incontestável, e do outro não se visualizam nem o que é necessário à manutenção da infraestrutura básica necessária a vida.

A falta de acesso a infraestrutura nos espaços urbanos só pode levar a produção, reprodução e acentuação da pobreza e da desigualdade socioespacial, quando essas estão relacionadas a ausência de saneamento básico, e também a presença de impactos ambientais, podem afetar a qualidade ambiental de uma localidade. Segundo Silveira (2006) esse processo existe tanto no campo como na cidade, mas nessa última é mais acentuada e evidente, assim como se distribui de maneira desigual por todo o território nacional.

Desse mesmo modo, Martinuci (2008) reafirma que nas cidades os lugares mais assistidos são normalmente os ocupados pelas classes sociais mais altas, de modo que este se configura como símbolo da dimensão social a qual o indivíduo se insere, assim a qualidade ambiental e a qualidade de vida são condicionados na mesma proporção.

Salles; Grigio; Silva (2013) alertam que os processos envoltos na urbanização e expansão das cidades geram inúmeros impactos não somente sociais, mas também ambientais e até mesmo sanitários. A ausência de rede coletora de esgoto, de coleta de resíduos sólidos, de acesso a saúde, entre outros serviços são alguns dos responsáveis por estes, isto, associado a ocupação acelerada que ocorre principalmente em áreas de vulnerabilidade agravam ainda mais os reflexos nocivos ao meio.

\section{Os resíduos sólidos urbanos}

Conforme Rodrigues (1998) a ausência de áreas adequadas para a disposição final dos resíduos sólidos e a pequena durabilidade dos produtos consumidos, agrava a problemática ambiental, sendo assim, é necessário buscar alternativas que diminuam a quantidade de rejeitos destinados aos aterros sanitários, buscando a manutenção e maior durabilidade dessas áreas. Uma das alternativas propostas pela autora é o reaproveitamento dos materiais passíveis de reciclagem.

Moraes (2011) aponta que os impactos ambientais em decorrência da disposição inadequada e através da decomposição e liberação do chorume acarretam danos em função da infiltração e escoamento, favorecendo a contaminação de solo, águas superficiais e subterrâneas.

Neste sentido, Peixoto (2005) assegura que a contribuição da coleta seletiva e da reciclagem é uma ação que colabora com a preservação do meio ambiente. Desse modo, ao realizar a destinação correta dos resíduos sólidos, concomitantemente contribuirá para a minimização dos impactos ambientais.

Entretanto, é cada vez mais claro que os problemas ambientais estão associados a questões políticas, ou seja, dependem de ações que visem soluções, pois nem todas as localidades são atendidas e tem acesso aos recursos de infraestrutura e serviços, como a 
coleta seletiva. Concordando com Leff (2001), as questões ambientais são políticas e, desse modo, não são nulas a interesses econômicos e políticos.

A alta produção de resíduos sólidos urbanos acarreta uma série de impactos, seja na poluição do ar a partir da liberação de gazes como $\mathrm{CO} 2$, na contaminação dos solos e consequentemente pelos processos de infiltração e escoamento das águas superficiais e subterrâneas. Esses problemas ocorrem a partir da ineficiência da gestão em que os resíduos são dispostos de maneira irregular, seja pela falta do serviço de coleta ou por questões culturais. Em detrimento dessa ação, outros problemas podem ser gerados como os de cunho sanitário com a proliferação de vetores de doenças.

No âmbito social os catadores buscam coletar materiais recicláveis para a comercialização e, às vezes, buscam por alimentos em locais de descarte irregular. Também se configura como um problema sanitário, pois essas áreas são insalubres no qual o indivíduo é exposto a vetores de doenças através de objetos e alimentos contaminados.

Os problemas envolvidos na gestão de resíduos sólidos são inúmeros e com certeza não estarão todos presentes nesse artigo, já que o objetivo é compreender como as ações de políticas públicas podem atuar para sanar esses conflitos. Conforme Secchi (2010), a função das políticas públicas é a resolução de problemas, os quais são definidos por ele como a diferença existente entre a real situação e a ideal de um determinado processo.

Em função dos problemas ambientais, Leff (2001) explana que estes se transformaram acima de tudo em assuntos políticos, ou seja, os impactos gerados em função da apropriação do meio se desdobram e ficam à mercê de interesses e, muitas vezes, sem garantias de soluções.

Para Leff (2001), a solução dos problemas ambientais fica condicionado aos interesses econômicos e de visualização política. Na mesma linha, Secchi (2010) argumenta que quando o problema passa a ser de cunho político, esse pode ser utilizado como matéria prima para gerar visibilidade de atividade em prol social.

De acordo com Secchi (2010), isso ocorre quando o problema adquire repercussão, e passam a despertar um determinado interesse de ação. Desse modo, os "atores" podem ser instituições, empresas, entre outros, podem ser públicos ou privados, e passam a demonstram interesses na solução das questões existentes a fim de sua promoção perante a sociedade.

Neste sentido, um problema ambiental, na maioria das ocorrências, só passa a ser analisado com a pretensão de resolução a partir do momento em que esse possa fornecer algum benefício para aquele que o promove. A gestão de resíduos sólidos passa pelo mesmo processo. Em síntese, a ineficiência das políticas públicas se apresenta de diversas maneiras nos espaços urbanos, os impactos ambientais podem estar ou não visíveis à população, porém apresentam riscos tanto ao meio ambiente quanto à saúde pública.

\section{Resultados e discussões}

Considerando as discussões a respeito da gestão dos resíduos sólidos, impactos ambientais e a importância das políticas públicas neste processo, apresenta-se o exemplo da cidade de Maringá, que conta com propostas para o programa de coleta seletiva desde 1994 e com início apenas em 2001. Entretanto, somente em 2017 passou a ter um plano municipal de gestão dos resíduos sólidos urbanos (PMGIRSU).

Segundo a Prefeitura do Município de Maringá (2017), até 2016 o programa atuou em $50 \%$ da cidade e a partir de 2017 a coleta foi expandida toda a malha urbana inclusive os distritos. Porém, devido as mudanças de gestão municipal e as diferentes visões sobre o tema o programa de coleta seletiva passou por várias modificações. 
A coleta seletiva atualmente é realizada de maneira híbrida por funcionários públicos e por terceirizados, mas já foi inteiramente pública e em certo momento também totalmente terceirizada. Entretanto, independentemente do método, os materiais coletados sempre foram destinados para as seis cooperativas parceiras (Cooperpalmeira, Coopermaringá, Coopernorte, Cooperambiental, Coopercanção e Coopervidros) da prefeitura do município.

A partir da análise dos dados da coleta seletiva e convencional, buscou-se verificar a sua efetividade e se as mudanças realizadas a partir de 2017 foram positivas. Para isso, comparou-se os dados da coleta seletiva e convencional (Gráfico 1).

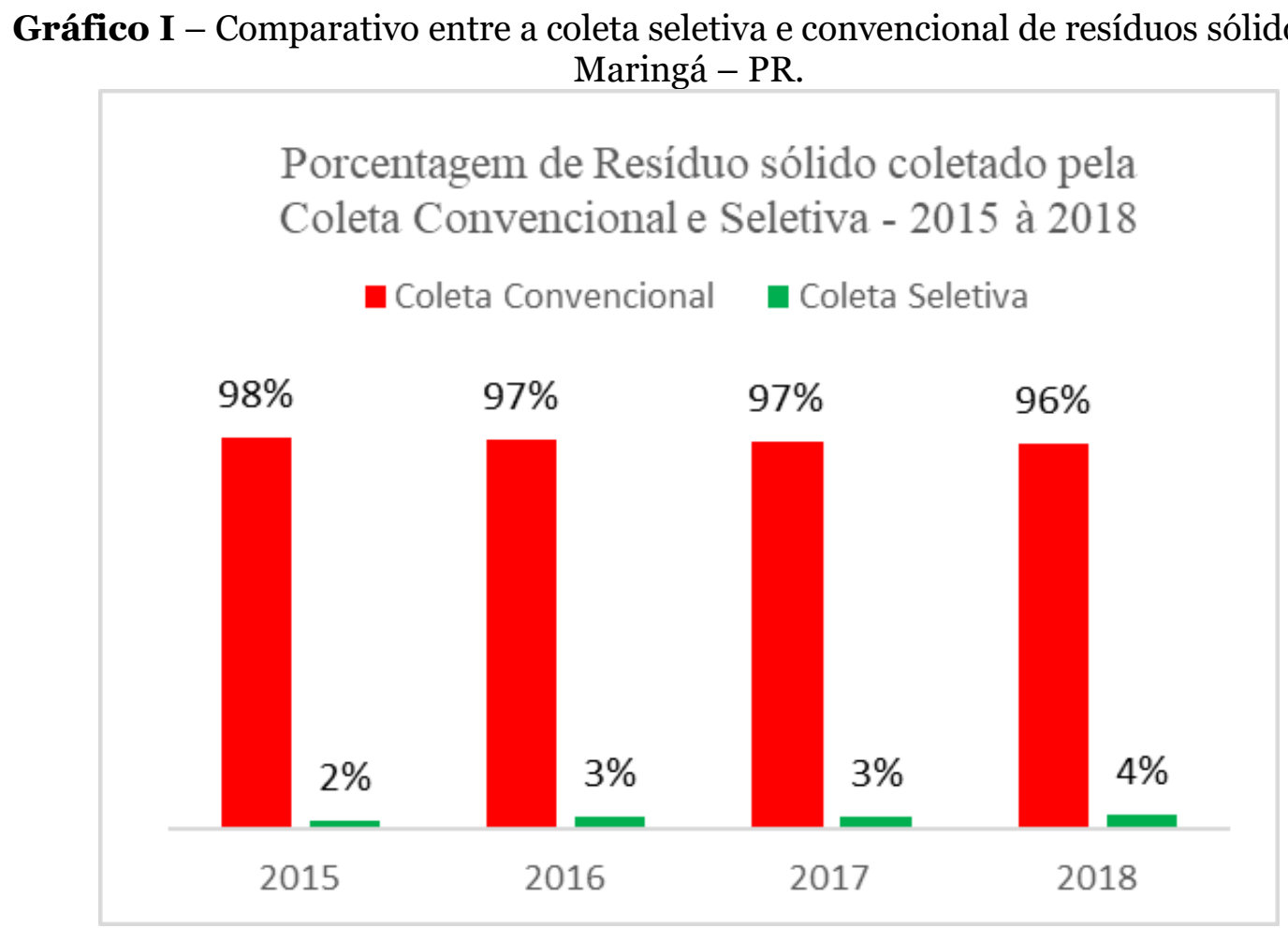

Elaboração: As autoras.

Em dados absolutos, constatou-se que foram coletados na cidade diariamente 762 gramas por habitante de resíduo sólido, desses apenas 28 gramas foram destinados para a reciclagem, ou seja, 734 gramas/habitante por dia foram destinados ao aterro sanitário.

A partir do Gráfico I, fica evidente que mesmo o programa de coleta seletiva tenha sido ampliado em 2017, não teve impacto significativo, e mesmo com as alterações realizadas na execução da coleta em 2018, teve um aumento de apenas $1 \%$. Portanto, fica evidente a ineficiência da coleta seletiva em Maringá, pois apenas 4\% de todo o resíduo sólido produzido na cidade foi coletado.

Além disso, identificou-se áreas de disposição irregular de resíduos na cidade, totalizando 31, a maioria concentradas em fundos de vale e terrenos baldios. Consideramos a disposição irregular de resíduos sólidos como uma falha na atuação do programa de coleta seletiva que deve incluir atividades de educação ambiental com a comunidade, tendo em vista que grande parte dos materiais encontrados nessas áreas eram passíveis de reciclagem. (Figura 2).

Kelly Cristina Rigoldi e Valéria Lima. A influência da gestão de resíduos sólidos na qualidade ambiental e nas desigualdades (...) Brazilian Geographical Journal: Geosciences and Humanities research medium, Ituiutaba, v. 11, n. 1, p. 85-96, jan./jul. 2020 
Figura 2 - Locais de disposição inadequada de resíduos sólidos em Maringá

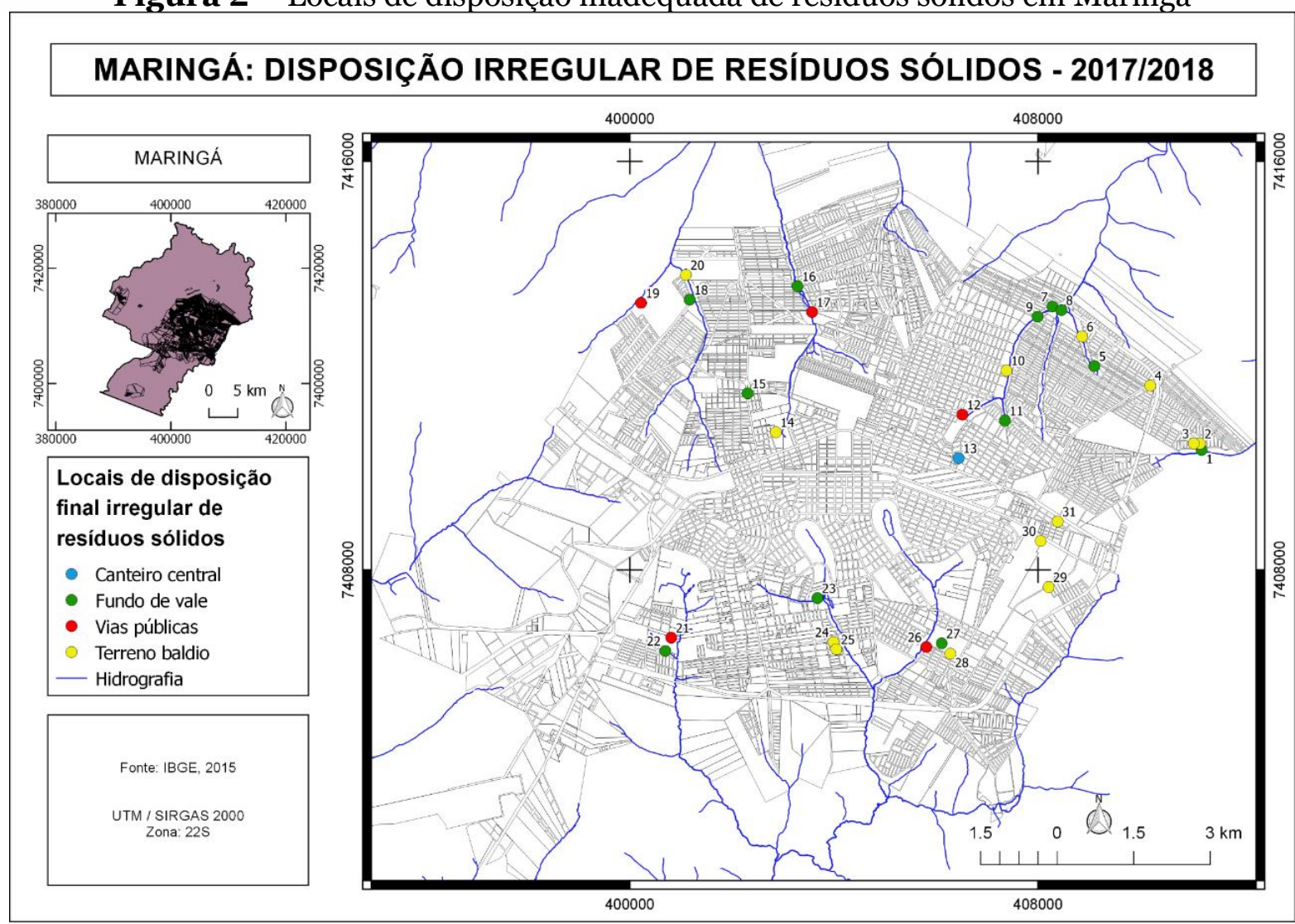

Elaboração: as autoras.

Nesse sentido, se os programas de educação ambiental e a atuação da coleta seletiva fossem efetivos, o resultado iria impactar diretamente na qualidade ambiental dessas áreas. (figuras 3, 4 e 5).

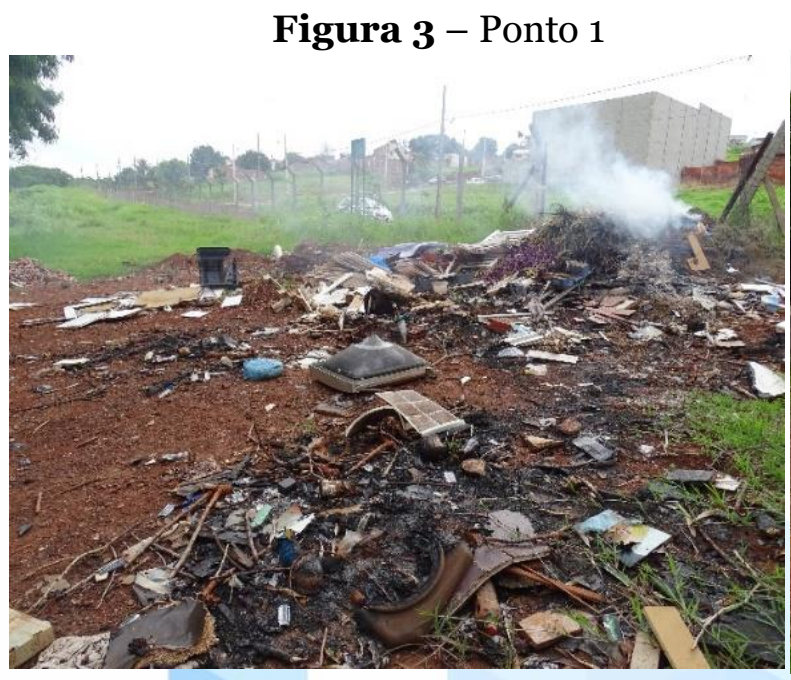

Fonte: Autoras, 2017.
Figura 4 - Ponto 2

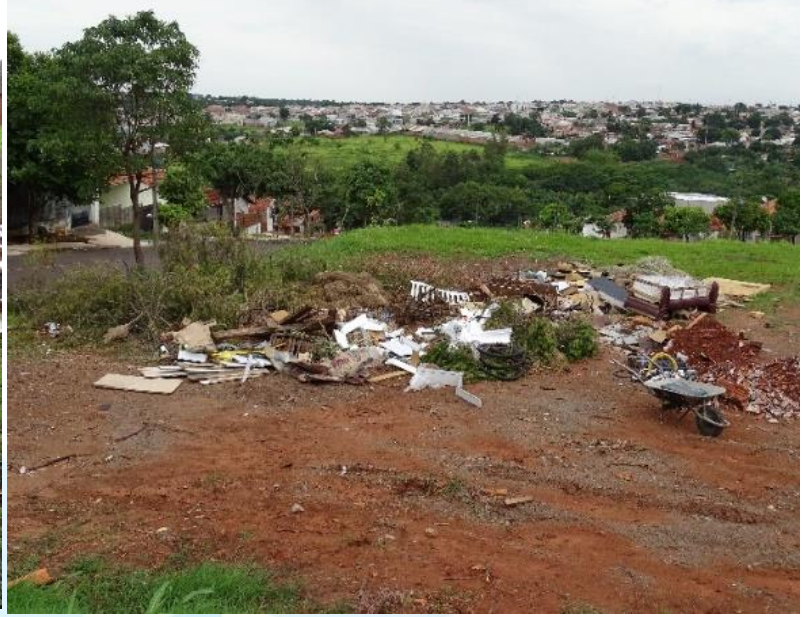

Fonte: Autoras, 2017.

Kelly Cristina Rigoldi e Valéria Lima. A influência da gestão de resíduos sólidos na qualidade ambiental e nas desigualdades (...) Brazilian Geographical Journal: Geosciences and Humanities research medium, Ituiutaba, v. 11, n. 1, p. 85-96, jan./jul. 2020. 


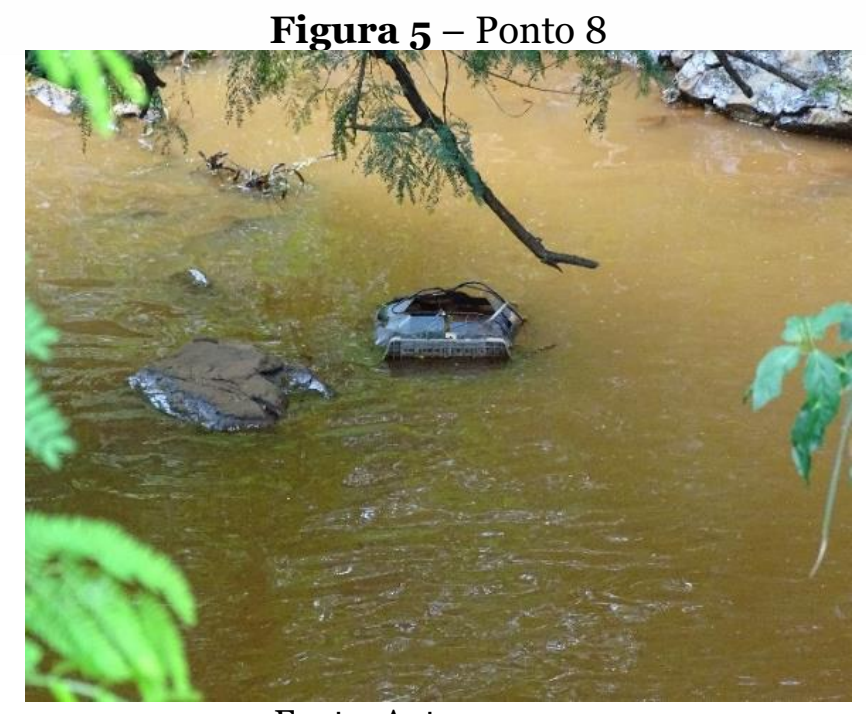

Fonte: Autoras, 2017.

Foram identificadas que as áreas de descarte irregular de resíduos sólidos, coincidem com as áreas de exclusão social da cidade de Maringá, as quais foram mapeadas e analisadas por Nunes (2016). As áreas de desigualdades sociais geralmente se apresentam com baixa qualidade ambiental e, conforme Guerra e Cunha (2006), é importante considerar o estudo das desigualdades territoriais durante a análise da qualidade ambiental urbana, apontando que os impactos ambientais estão associados a condição de renda de uma sociedade, portanto, indicam que as áreas mais afetadas ambientalmente são também aquelas que possuem menor acesso a infraestrutura e, consequentemente, as que alocam a população de baixa renda das cidades.

Nesse sentido, verificou-se que as áreas mais afetadas são as periféricas, tanto pela disposição irregular de resíduos como por áreas de exclusão social. (Figura 4).

Na porção norte da cidade as áreas mais atingidas pelo descarte irregular coincidem com a condição de alta exclusão social, de modo que as vulnerabilidades sociais e ambientais se sobrepõem, acentuando as desigualdades socioespaciais, e consequentemente interferindo a qualidade ambiental a de vida da população.

De acordo com Rosa et al (2010) o descarte irregular de resíduos sólidos, traz diversos impactos, sendo eles de cunho ambiental, social e sanitário, gerando riscos à saúde, a partir da proliferação de vetores transmissores de doenças como, Leishmaniose, Leptospirose, Hepatite, Dengue e Alergias.

A ineficiência das políticas públicas, se manifestam de diversas maneiras nas paisagens urbanas, em relação a gestão de resíduos e vale ressaltar que essas ferramentas não atuam apenas na disponibilidade da atuação da coleta seletiva, mas também em programas de educação ambiental, os quais são fundamentais para a sensibilização da população e para o funcionamento adequado dos programas. Entretanto, os problemas decorrentes da ineficácia dessas ações, podem não estar visíveis para a população. Nesse caso, a ineficácia dessas políticas estará vinculada também na acentuação das desigualdades socioespaciais, onde as áreas de vulnerabilidade social se correlacionam com as de baixa qualidade ambiental. 
Figura 4 - Exclusão social e disposição irregular de resíduos sólidos

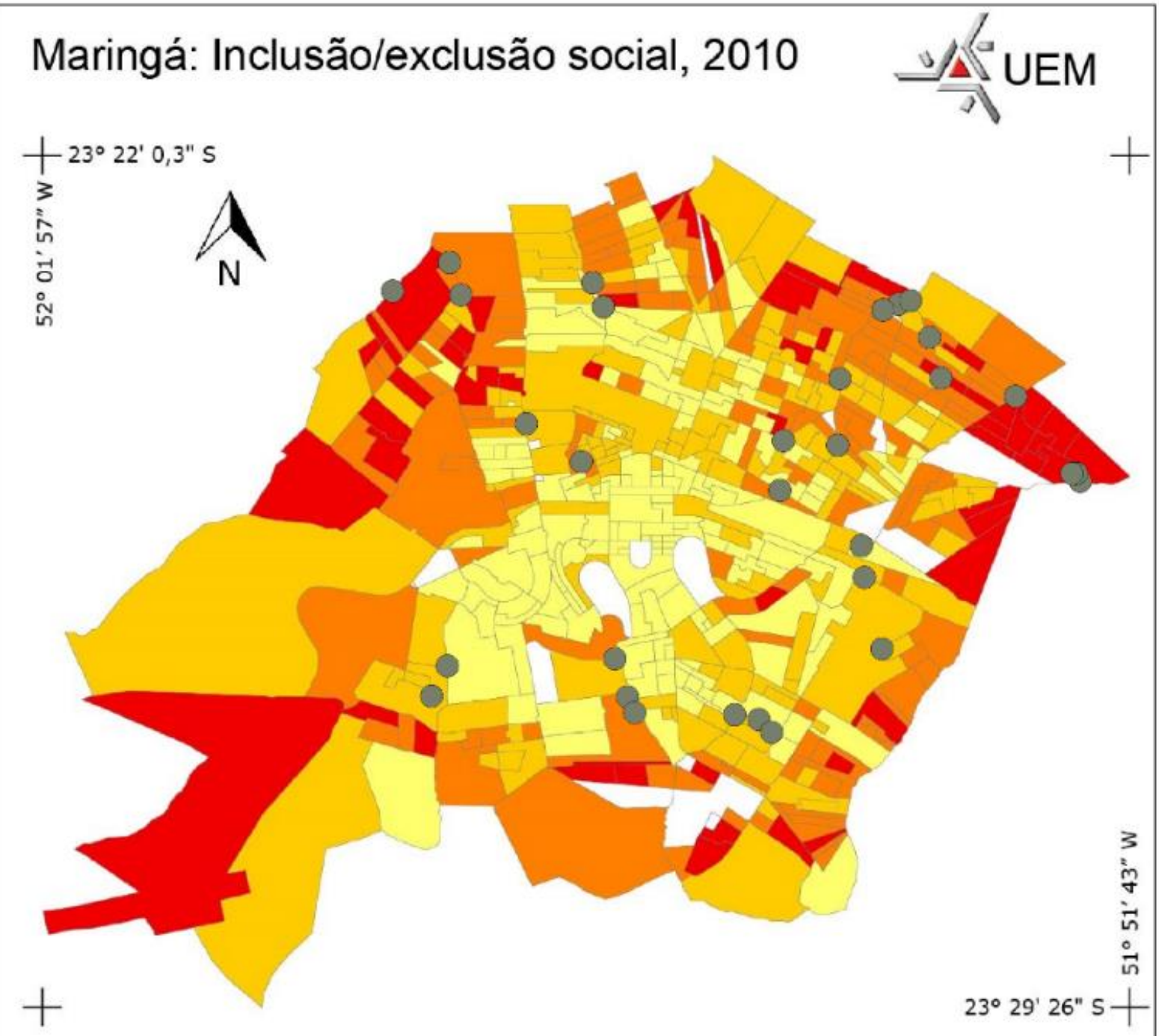

Sintese dos indicadores (frequência)

Alta exclusão social

Média exclusão social

Baixa exclusão social

Inclusão social

Ausência de informação

Pontos de descarte irregular de resíduos

Eleborado com Philcarto *22/12/2016 20:00:27* http://philcarto free ff

Datum: Sirgas, 2000

Fonte de dados: IBGE - Censo

Demográfico 2010

Org: Livia Fiorillo Nunes, 2016

Escala gráfrica aproximada

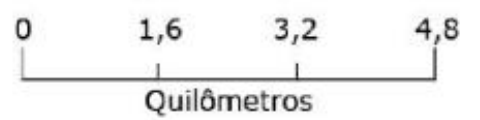

Fonte: NUNES (2016). Adaptado pelas autoras, 2019.

Kelly Cristina Rigoldi e Valéria Lima. A influência da gestão de resíduos sólidos na qualidade ambiental e nas desigualdades (...) Brazilian Geographical Journal: Geosciences and Humanities research medium,

Ituiutaba, v. 11, n. 1, p. 85-96, jan./jul. 2020 


\section{Conclusão}

Diante das análises dos dados disponibilizados pela SEMUSP, conclui-se que a coleta seletiva em Maringá ainda é inexpressiva. Mesmo com a implantação do PMGIRSU (2017) e das mudanças realizadas a partir de 2017, o aumento foi pequeno, apontando falhas na execução do plano de gestão.

A partir da realização dos trabalhos de campo que contribuiram para as análises, levando em consideração a organização socioespacial da cidade, foi possível a identificação da disposição irregular de resíduos, onde contastou-se que nas periferias e nas áreas de vulnerabilidade social, é comum a disposição irregular de resíduos sólidos.

A qualidade ambiental urbana em relação aos resíduos sólido em Maringá é impactada negativamente pela presença de áreas de disposição irregular. Além disso, o Programa de coleta seletiva na cidade ainda não é eficaz, e por isso consideramos necessária a atuação de políticas públicas que visem aprimorar e tornar este efetivo.

\section{Referências}

DANTAS, F. D. M.; DANTAS, L. D. M.; DIAS, L. S. O. F. O impacto da expansão urbana para o meio ambiente. In: $\mathrm{V}$ congresso em desenvolvimento social: estado, meio ambiente e desenvolvimento, 2016, Montes Claros. Anais do V congresso em desenvolvimento social: estado, meio ambiente e desenvolvimento: Montes Claros: 2016, p. 5-12.

GUERRA, A. J. T. e CUNHA, S.B. Impactos Ambientais Urbanos no Brasil. Rio de Janeiro: Bertrand Brasil, 2006.

HARVEY, D. O direito a cidade. Tradução por: PINHEIRO, J. Lutas sociais: São Paulo, n. 29, 2012, p. 73-89.

IPARDES - Instituto Paranaense de Desenvolvimento Econômico e Social. Relação dos municípios do estado ordenados segundo as mesorregiões e as microrregiões geográficas do IBGE. Paraná, 2012, 8 p.

LEFF, E. Saber Ambiental: sustentabilidade, racionalidade, complexidade, poder. Rio de janeiro: Vozes, 2001.

MARQUES, J. R. Meio ambiente urbano. 1 ed. Rio de Janeiro: Forense Universitária, 2005, $233 \mathrm{p}$.

MARTINUCI, O. da S. Circuitos e modelos da desigualdade social intra-urbana. 2008. 146 f. Dissertação (Mestrado em Geografia), Universidade Estadual Paulista, Faculdade de Ciências e Tecnologia, Presidente Prudente.

MENDONÇA, Francisco. Geografia e meio ambiente. 9 ed. São Paulo: Contexto, 2012, 80 p..

socioambientais urbanos. Curitiba: UFPR, 2004.

(org); MONTEIRO, Carlos A. F. et al. Impactos

NUNES, L. F. Mapeamento da inclusão/exclusão social da cidade de Maringá-PR. 2017. Trabalho de conclusão de curso. Universidade Estadual de Maringá, Maringá 2016. 
PEIXOTO, K. CAMPOS, V. B. G., D'AGOSTO, M. A. A coleta seletiva e a redução de resíduos sólidos. Rio de janeiro: Instituto Militar de Engenharia. 2005

PMM - PREFEITURA DO MUNICÍPIO DE MARINGÁ. Plano municipal de gestão integrada de resíduos sólidos urbanos. Maringá, 2017, 498 p.

QGIS Development Team (2019). QGIS Geographic Information System. Open Source Geospatial Foundation Project. Disponível em: <http://qgis.osgeo.org>. Acesso em: 16 set. 2019

RODRIGUES, Arlete Moysés. Produção e consumo do e no espaço: Problemática ambiental urbana. São Paulo: Hucitec, 1998.

SALLES, M. C. T.; GRIGIO, A. M.; SILVA, M. R. F. da. Expansão urbana e conflito ambiental: uma descrição da problemática do município de Mossoró, RN - brasil. Soc. \& Nat., Uberlândia, 25 (2): 281-290, mai/ago/2013.

SANTOS, M. A natureza do espaço: técnica e tempo. Razão e emoção. 4 ed. São Paulo: Editora da Universidade de São Paulo, 2006.

SANTOS, M. A urbanização brasileira. 5 ed. São Paulo: Editora da Universidade de São Paulo, 2009.

SECCHI, L. Políticas Públicas: Conceitos, Esquemas de Análise, Casos Práticos. São Paulo: Cengage Learning, 2010.

SILVEIRA, M. L. Por que há tantas desigualdades sociais no Brasil? Um panorama da riqueza e da pobreza brasileira. In: ALBUQUERQUE, E. S. de. (Org.). Que País é esse? Pensando o Brasil contemporâneo. São Paulo: Globo, 2006, 151 p.

Recebido em: 11/12/2019 Aprovado para publicação em: 22/06/2020 